BNL-75351-2006-CP

\title{
Measurements of the Field Quality in Superconducting Dipoles at High Ramp Rates
}

\author{
A. Jain, J. Escallier, G. Ganetis, W. Louie, A. Marone, \\ R. Thomas, P. Wanderer
}

Presented at the $19^{\text {th }}$ International Conference on Magnet Technology

Genova, Italy

September 18-23, 2005

December 16, 2005

\author{
Superconducting Magnet Division \\ Brookhaven National Laboratory \\ P.O. Box 5000 \\ Upton, NY 11973-5000 \\ www.bnl.gov
}

\begin{abstract}
Notice: This manuscript has been authored by employees of Brookhaven Science Associates, LLC under Contract No. DE-AC02-98CH10886 with the U.S. Department of Energy. The publisher by accepting the manuscript for publication acknowledges that the United States Government retains a non-exclusive, paid-up, irrevocable, world-wide license to publish or reproduce the published form of this manuscript, or allow others to do so, for United States Government purposes.
\end{abstract}

This preprint is intended for publication in a journal or proceedings. Since changes may be made before publication, it may not be cited or reproduced without the author's permission. 


\section{DISCLAIMER}

This report was prepared as an account of work sponsored by an agency of the United States Government. Neither the United States Government nor any agency thereof, nor any of their employees, nor any of their contractors, subcontractors, or their employees, makes any warranty, express or implied, or assumes any legal liability or responsibility for the accuracy, completeness, or any third party's use or the results of such use of any information, apparatus, product, or process disclosed, or represents that its use would not infringe privately owned rights. Reference herein to any specific commercial product, process, or service by trade name, trademark, manufacturer, or otherwise, does not necessarily constitute or imply its endorsement, recommendation, or favoring by the United States Government or any agency thereof or its contractors or subcontractors. The views and opinions of authors expressed herein do not necessarily state or reflect those of the United States Government or any agency thereof. 


\title{
Measurements of the Field Quality in Superconducting Dipoles at High Ramp Rates
}

\author{
Animesh Jain, John Escallier, George Ganetis, Wing Louie, Andrew Marone, Richard Thomas, \\ Peter Wanderer
}

\begin{abstract}
Several recent applications of superconducting magnets require the magnets to be operated at high ramp rates and at frequencies of several Hertz. Brookhaven National Laboratory (BNL) has recently designed and built prototypes of superconducting dipole magnets that can be ramped at a fairly high rate (1 $\mathrm{T} / \mathrm{s}$ or more). For accelerator applications, it is also crucial that the magnets maintain good field quality even at high ramp rates. In order to characterize the field quality of magnets at high ramp rates, a measurement system consisting of 16 printed circuit tangential coils has been developed. The coil system is held stationary while the magnet is ramped. This paper describes the techniques used for the measurements and data analysis, and presents the results of measurements at ramp rates of up to $4 \mathrm{~T} / \mathrm{s}$ in a prototype dipole built at BNL for GSI.
\end{abstract}

Index Terms-Superconducting accelerator magnets, magnetic field measurement.

\section{INTRODUCTION}

$\mathrm{T}$ $\mathrm{HE}$ field quality in accelerator magnets is expressed in terms of field harmonics. Magnetic field harmonics in nearly all accelerator magnets are measured with rotating coils with a period of several seconds. Such measurement systems are ideally suited for static fields. One may extend the capabilities of the rotating coils to slowly varying fields $(<0.1 \mathrm{~T} / \mathrm{s})$ using an improved data analysis scheme [1]. This has been used successfully to study some dynamic effects in superconducting magnets, such as time decay and snapback. For higher ramp rates, the approximations used in the analysis

Manuscript received September 20, 2005. This work was supported in part by the U.S. Department of Energy under contract number DE-AC02$98 \mathrm{CH} 10886$ and in part by Geselischaft für Schwerionen Physik (GSI). This manuscript has been authored by Brookhaven Science Associates, LLC under Contract No. DE-AC02-98CH1-886 with the U.S. Department of Energy. The United States Government retains, and the publisher, by accepting the article for publication, acknowledges, a worldwide license to publish or reproduce the published form of this manuscript, or allow others to do so, for the United States Government purposes.

The authors are with the Superconducting Magnet Division, Brookhaven National Laboratory, PO Box 5000, Upton NY 11973. Their other contact information is:

A. Jain (phone: 631-344-7329, fax: 631-344-2190, email: jain@bnl.gov).

J. Escallier (escallie@bnl.gov)

G. Ganetis (ganetisl@bnl.gov)

W. Louie (louie@bnl.gov)

A. Marone (andym@bnl.gov)

R. Thomas (thomas1@bnl.gov)

P. Wanderer (wanderer@bnl.gov) are no longer valid and it is difficult to extract useful harmonic information from conventional rotating coil systems.

A system with non-rotating probes does not have the characteristic time scale limitations suffered by rotating systems. In order to obtain field harmonics, one needs to use an array of several probes. One may use an array of active probes, such as Hall probes [2], but careful intercalibration of the probes is necessary and nonlinearities and other effects may limit the accuracy and dynamic range of such a system.

In order to carry out field quality measurements at very high ramp rates, we have recently developed a new system consisting of an array of 16 pickup coils [3]. Some early results of measurements of the sextupole term in a superconducting dipole ramped at $3 \mathrm{~T} / \mathrm{s}$ were presented in an earlier paper [4]. In this paper, we present results of more extensive measurements made in a superconducting dipole at ramp rates of 1.5 to $4 \mathrm{~T} / \mathrm{s}$. A slightly improved data acquisition and analysis scheme to overcome some difficulties in the earlier measurements is also described.

\section{EXPERIMENTAL DETAILS}

\section{A. Measurement System}

The measurement probe consists of a set of 16 tangential coils mounted on a cylindrical coil form, as shown schematically in Fig. 1. The coils are made of 10-layer printed circuit boards with six turns per layer. The windings are $300 \mathrm{~mm}$ long, and are located at a nominal radius of $26.8 \mathrm{~mm}$, with a nominal opening angle of 17.5 degrees.

The voltage signals from all the windings are fed to custom built programmable gain amplifiers (up to 100X). The outputs of the amplifiers are recorded using 16-bit ADCs. It would be desirable to use high-resolution voltmeters, but an ADC based system was built to reduce the cost. More details of the probe construction and the data acquisition system can be found in references [3] and [4].

The probe can also be used in a rotating mode to measure DC fields. This feature is used to measure the field quality under DC excitation, which provides a baseline for comparison with the data obtained under ramping conditions. The rotating mode is also used to measure the initial values of harmonics at constant current just before the start of a ramp, needed for data analysis. In the case of measurements while ramping, the probe is held stationary, and the induced voltages 
well as the instantaneous magnet current, are sampled simultaneously at a suitable rate, which can be up to $10 \mathrm{kHz}$. For the present work, the sampling rates varied from $48 \mathrm{~Hz}$ to $105 \mathrm{~Hz}$, and were chosen to provide approximately 256 data points for every cycle of one up and down ramp.

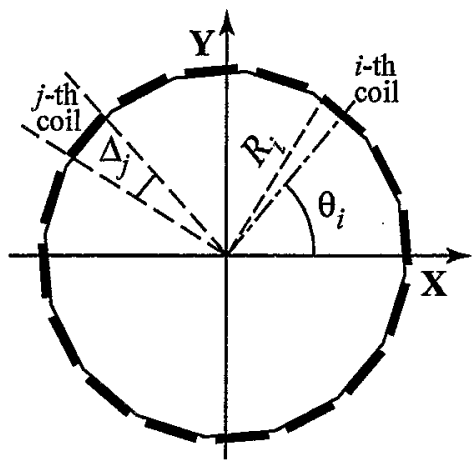

Fig. 1 Schematic showing a cross section of the probe, and the relevant geometric parameters of each coil.

\section{B. Magnet Details}

The magnet used for measurements reported in this paper is a $1 \mathrm{~m}$ long prototype dipole built as part of the R\&D for the Facility for Antiproton and Ion Research (FAIR) at Gesellschaft für Schwerionen Physik (GSI). This magnet has an $80 \mathrm{~mm}$ inner diameter coil, and is designed for operation at high ramp rates. A stainless steel cored cable and G11 coil wedges are some of the unique features of this magnet in order to minimize eddy currents. More details of the magnet construction and some test results can be found in [4]-[6].

This magnet was first tested for operation up to $4 \mathrm{~T} / \mathrm{s}$ with a laminated silicon steel yoke around the collared coil. Preliminary measurements of field quality were also made [4] with the measurement system described above. Subsequently, the iron yoke was removed in order to study eddy current losses in the coil. Field measurements presented in this paper were made during cold tests of this magnet without the yoke.

\section{Ramp profiles}

The magnet was first cycled three times between $0.1 \mathrm{kA}$ and $7 \mathrm{kA}(3.2 \mathrm{~T})$ at the intended ramp rate to set the history. All field measurements were started with the magnet at a fixed current of $0.1 \mathrm{kA}$. The DC field was first measured with the probe operated in a rotating mode. The probe was then held stationary while the magnet was ramped up and down between $0.1 \mathrm{kA}$ and $7 \mathrm{kA}$. A ramp up consisted of an initial dwell at $0.1 \mathrm{kA}$ of $0.6 \mathrm{~s}$, a quadratic acceleration phase, a linear (constant ramp rate) segment, and finally a quadratic deceleration phase leading to $7 \mathrm{kA}$ flat top. The parameters of the acceleration and deceleration phases were adjusted such that each phase lasted for about $10 \%$ of the total ramp time. After a $0.1 \mathrm{~s}$ dwell at $7 \mathrm{kA}$, a similar profile was used for ramping down to $0.1 \mathrm{kA}$, which completed one ramp cycle. In a typical measurement sequence, four such ramp cycles were repeated one after the other, while continuously sampling the voltage outputs from the 16 coils. Such a measurement provides four sets of up and down ramp harmonics in the entire current range, thus allowing a cycle-to-cycle comparison of the data. Finally, the entire sequence of four up and down ramp cycles was measured multiple times to check the reproducibility of the measurements.

\section{DATA ANALYSIS}

\section{A. Basic formalism}

The relevant geometric parameters of the coils are shown in Fig. 1. The X-axis of the coordinate system is defined by an index pulse from an angular encoder in the rotating mode. The angular positions, $\theta_{i}$, of all the coils are determined in this coordinate system by rotating the coil in a reference dipole field. Similarly, the radii, $R_{i}$, and the opening angles, $\Delta_{i}$, are determined by rotating the probe in known dipole and quadrupole fields. A system has been developed to reproducibly align (within $\sim 0.1 \mathrm{mrad}$ ) the probe with this coordinate system in the non-rotating mode.

The voltage induced in the $i$-th coil in the stationary mode results from a time variation of the flux linked to the coil at time $t$, and is given by

$$
\begin{aligned}
V_{i}(t) & =\sum_{n=1}^{\infty} \frac{2 N_{i} L_{i} R_{r e f}}{n}\left(\frac{R_{i}}{R_{r e f}}\right)^{n} \sin \left(\frac{n \Delta_{i}}{2}\right) \\
& \times\left[\dot{B}_{n}(t) \sin \left(n \theta_{i}\right)+\dot{A}_{n}(t) \cos \left(n \theta_{i}\right)\right]
\end{aligned}
$$

where $L_{i}$ is the length of the windings, and $N_{i}$ is the number of turns in the $i$-th coil. $B_{n}(t)$ and $A_{n}(t)$ are the normal and skew $2 n$-pole field harmonic components respectively at a reference radius of $R_{r e f}$. The dot over $B_{n}(t)$ and $A_{n}(t)$ denotes the time derivative. The 16 coils in the probe span the complete azimuthal range and the voltage outputs can be used to extract the time derivatives of the normal and skew terms. If the geometric parameters of all the coils were identical, and the coils were equally spaced in angular position, then $\dot{B}_{n}(t)$ and $\dot{A}_{n}(t)$ could be obtained by a simple Fourier analysis. In practice, all the coils are not identical (although are very similar), and the angular positions are not exactly equispaced. This, a Fourier analysis does not provide an accurate solution in this situation.

For the analysis of our data, we have obtained the time derivatives $\dot{B}_{n}(t)$ and $\dot{A}_{n}(t)$ by a linear least squares fitting to all the 16 voltage signals at any given instant. The summation in (1) is truncated at a chosen maximum harmonic, $n_{\max }$. With 16 signals, $n_{\max }$ may be up to 8 (16-pole). However, that leaves no redundancy in the data and the 16pole terms were found to be dominated by the noise in the signals. Consequently, $n_{\max }$ was reduced to 7 (14-pole). The analysis assumes that any contribution from terms higher than 14-pole is negligible. In order to test the stability of the solution, we have also carried out analysis with $n_{\max }=6$, which gave almost identical results. 
The harmonic components at any time, $t$, are obtained by integrating the time derivatives:

$$
B_{n}(t)=B_{n}(0)+\int_{0}^{t} \dot{B}_{n}(t) d t, A_{n}(t)=A_{n}(0)+\int_{0}^{t} \dot{A}_{n}(t) d t
$$

The initial values of the harmonics, $B_{n}(0)$ and $A_{n}(0)$, are determined using the same probe in a rotating mode just before the magnet is ramped.

\section{B. Offset correction}

Since a constant voltage in a given coil implies a constant time derivative of the flux linked through that coil, any offsets in the measured signals will translate into offsets in the quantities $\dot{B}_{n}(t)$ and $\dot{A}_{n}(t)$. Even small errors in these time derivatives may result in a significant drift of the computed harmonics after integration. As a result, the relative value of a harmonic at a given current on the down ramp (which is measured at a later time) may be shifted with respect to the up ramp value. Such drifts lead to large run-to-run variations in the measured hysteresis. Even though we have followed a procedure to characterize the offset and gain of each channel, we do see noticeable drifts in harmonics in nearly all runs.

Fig. 2 shows an example of the drift in the computed normal sextupole in four successive ramps from $0.1 \mathrm{kA}$ to $7 \mathrm{kA}$ at $6.6 \mathrm{kA} / \mathrm{s}(3 \mathrm{~T} / \mathrm{s})$. The triangles and circles mark the values of the sextupole at fixed currents of $1.5 \mathrm{kA}$ and $6 \mathrm{kA}$ respectively on the up ramp of the four cycles. A drift with time can be clearly seen. Assuming that the harmonics at a fixed current in the successive cycles should really be the same, one can compute the offsets in all the $\dot{B}_{n}(t)$ and $\dot{A}_{n}(t)$. The offsetcorrected harmonics, $B_{n}^{\text {cor }}(t)$ and $A_{n}^{\text {cor }}(t)$ are then given by

$$
B_{n}^{\text {cor }}(t)=B_{n}(t)-\dot{B}_{n}^{\text {off }} \cdot t \text { and } A_{n}^{\text {cor }}(t)=A_{n}(t)-\dot{A}_{n}^{\text {off }} \cdot t
$$

where $B_{n}(t)$ and $A_{n}(t)$ are the uncorrected $2 n$-pole normal and skew harmonics and $\dot{B}_{n}^{\text {off }}$ and $\dot{A}_{n}^{\text {off }}$ are the offsets computed from the slopes of straight line fits to the data at a

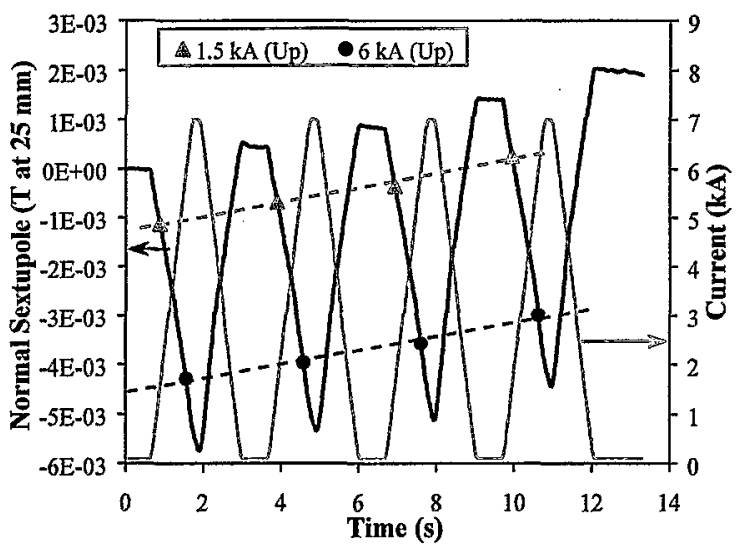

Fig. 2 The current ramp profile showing the four up and down cycles, and the computed normal sextupole term at $25 \mathrm{~mm}$ reference radius. The symbols mark the values of the harmonic measured at a fixed current on the up ramp in the four successive cycles. The dashed lines represent straight line fits to the data points at a given current. fixed current. We have used an average of 22 offset values derived from a set of 11 currents chosen in the range of $2 \mathrm{kA}$ to $4 \mathrm{kA}$ (in the linear ramp region) for both the up and the down ramps. For this technique of offset correction to work, it is clear that a measurement sequence must consist of at least two identical ramps. Use of more than two ramps (e.g. four in the present work) allows one to also better estimate the uncertainty in the measurements.

\section{RESULTS}

We have measured the field harmonics in the prototype dipole magnet ramped from $0.1 \mathrm{kA}$ to $7 \mathrm{kA}(3.18 \mathrm{~T})$ at ramp rates of 3.3 to $8.8 \mathrm{kA} / \mathrm{s}(1.5$ to $4 \mathrm{~T} / \mathrm{s})$. Three measurements, each comprising four successive ramp cycles, were made for each ramp rate. There is some uncertainty $(\sim 10 \%)$ in the offset corrections determined from various cycles within a run, leading to some cycle-to-cycle variation in the computed harmonics. This is perhaps caused by noise in the data acquisition system. The harmonics, when averaged over all the four cycles of a run, show better run-to-run agreement.

Fig. 3 shows the offset corrected normal and skew sextupole terms as a function of current, measured in the four successive cycles at a ramp rate of $2 \mathrm{~T} / \mathrm{s}(4.4 \mathrm{kA} / \mathrm{s})$. The cycle-to-cycle variation is evident, particularly at lower excitation. The rather large geometric sextupole is present as the coil cross section was not optimized for the oversized cable. Fig. 4 shows a similar plot for the decapole, which is the next allowed term. It is noteworthy that no saturation of the harmonics is seen at higher fields since the magnet does not have an iron yoke. In an earlier test of this magnet with iron yoke, the sextupole had shown significant saturation [4] consistent with calculations.

The variation of the normal sextupole term as a function of current is shown in Fig. 5 for all the ramp rates that were measured. The DC data were obtained using the same measurement system in a rotating mode. All the fast ramp data shown are the average values of results from three separate measurement sets. The effect of eddy currents is seen more clearly in a plot of the measured differences between the down ramp and the up ramp harmonics, as shown in Fig. 6. The

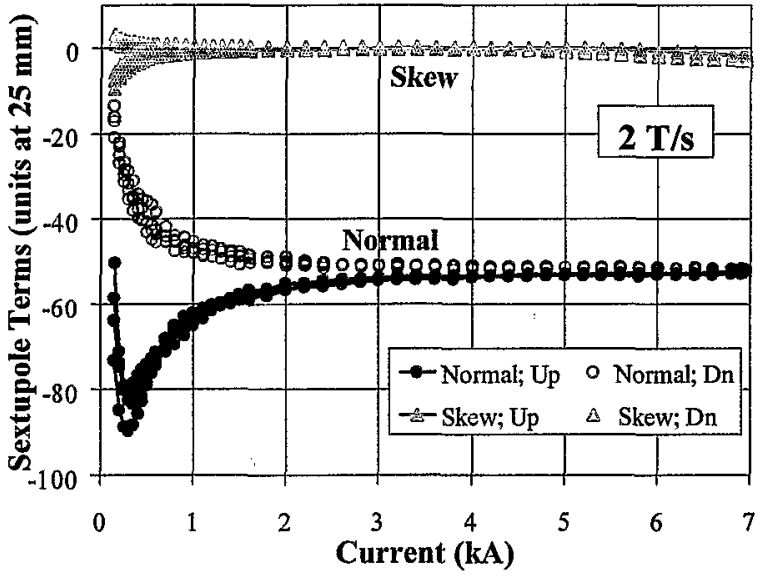

Fig. 3 The offset-corrected normal and skew sextupole terms as a function of current measured in the four ramp cycles of a single run at $2 \mathrm{~T} / \mathrm{s}$. The values are expressed in dimensionless "units" of $10^{-4}$ of the dipole term. 


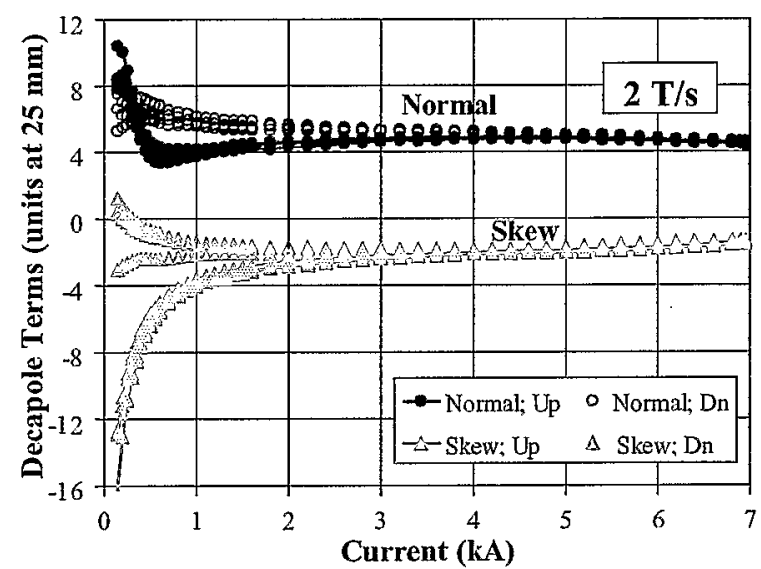

Fig. 4 The offset-corrected normal and skew decapole terms as a function of current measured in the four ramp cycles of a single run at $2 \mathrm{~T} / \mathrm{s}$. The values are expressed in dimensionless "units" of $10^{-4}$ of the dipole term.

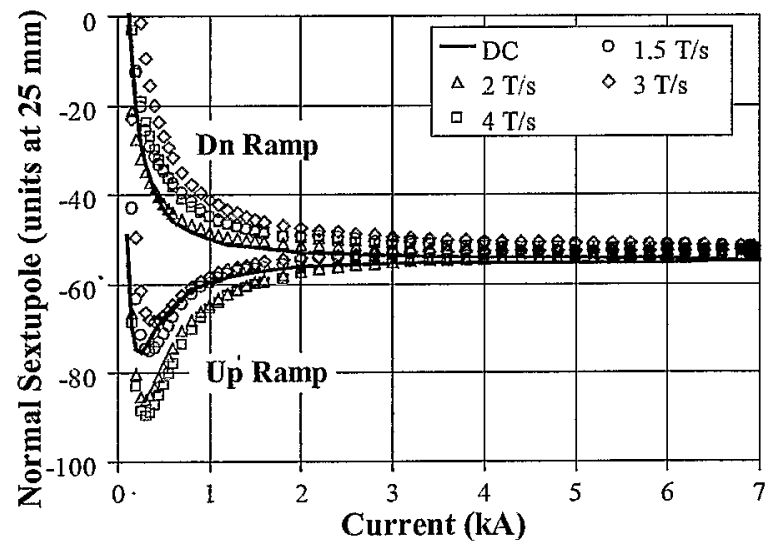

Fig. 5 The variation of normal sextupole term as a function of current at various ramp rates. The $\mathrm{DC}$ data were obtained using the same measurement system in a rotating mode. All the fast ramp data shown are the average values from three separate measurement sets.

ramp and the up ramp harmonics, as shown in Fig. 6. The absolute values in Tesla are plotted to prevent normalization by large fields at higher currents. The hysteresis at a fixed current of $2 \mathrm{kA}(0.91 \mathrm{~T})$ is summarized in Fig 7 (sextupole) and Fig. 8 (decapole). The results from various runs agree with a standard deviation of less than 1 unit at this field. The lines in Figs. 7 and 8 are linear fits to the data. The intercept is constrained to the $\mathrm{DC}$ value, which is obtained in the rotating

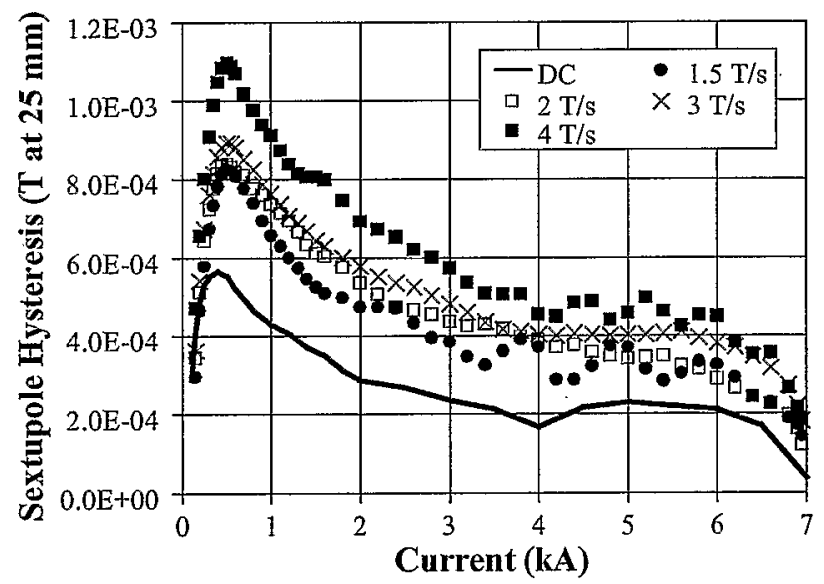

Fig. 6 Difference between the down ramp and the up ramp values of the normal sextupole term as a function of current at various ramp rates.

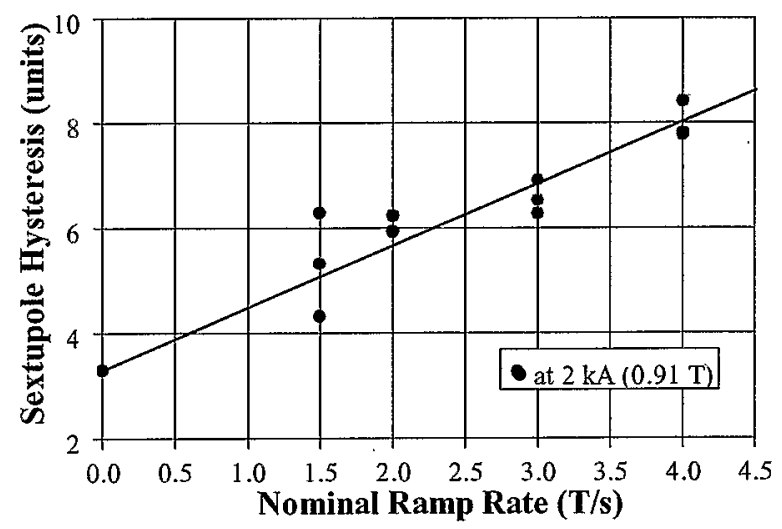

Fig. 7 Sextupole hysteresis at $2 \mathrm{kA}(0.91 \mathrm{~T})$ as a function of ramp rate.

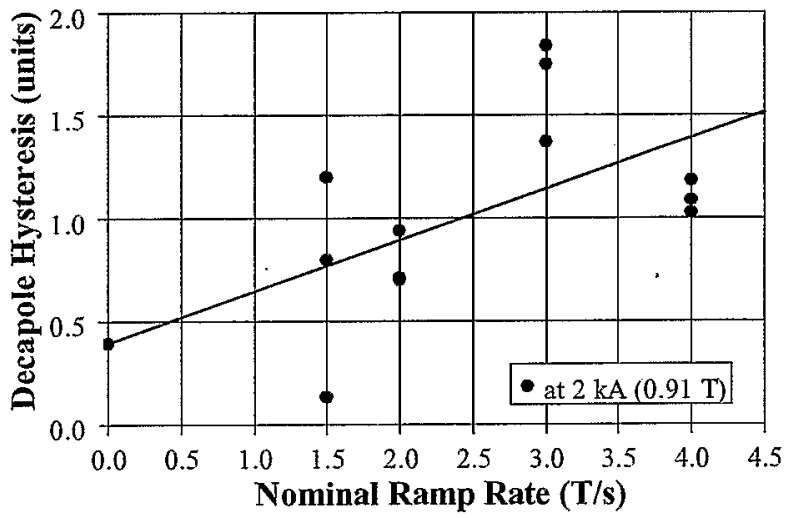

Fig. 8 Decapole hysteresis at $2 \mathrm{kA}(0.91 \mathrm{~T})$ as a function of ramp rate.

mode and has much smaller uncertainty. The slopes are given by $1.2 \mathrm{unit} /(\mathrm{T} / \mathrm{s})$ for the sextupole and $0.25 \mathrm{unit} /(\mathrm{T} / \mathrm{s})$ for the decapole. The eddy current induced harmonics can be taken to be half of these values. The 14-pole term is below 1 unit, and showed no measurable ramp rate effect.

\section{ACKNOWLEDGMENT}

We are grateful for the contributions of our technician staff, J. Cintorino, S. Dimaiuta, G. Herbst, W. Mc Keon, A. Sauerwald, and D. Sullivan.

\section{REFERENCES}

[1] A. Jain, "Recent magnetic measurement activities at BNL," Proc. $12^{\text {th }}$ International Magnetic Measurement Workshop, Grenoble, France, Oct. $1-4,2001$. Available on-line at $\mathrm{http} / /$ www.esrf.fr/conferences/proceedings/IMMW12/present.htm

[2] M. Haverkamp, L. Bottura, E. Benedico, S. Sanfilippo, B. ten Haken, H.H.J. ten Kate, "Field Decay and Snap-back Measurements Using a Fast Hall Plate Detector", IEEE Trans. Appl. Sup., 12 (1), 86-89, 2002.

[3] P. Wanderer et al., "Development of a precise magnetic field measurement system for fast-changing magnetic fields," Proc. $18^{\text {th }}$ Intl. Conf. on Magnet Technology, Morioka, Japan, Oct. 20-24, 2003, in IEEE Trans. Appl. Superconductivity, Vol. 14, No.2, June 2004, pp. $1826-9$.

[4] A. Jain, et al., "Magnetic field measurements for fast changing magnetic fields,: Proc. Applied Superconductivity Conference 2004, October 3-8, 2004, Jacksonville, FL, in IEEE Trans. Appl. Superconductivity, Vol. 15, No.2, June 2005, pp. 1221-4.

[5] G. Moritz, "Fast-pulsed SC magnets," Proc. 9th European Particle Accelerator Conference, Lucerne, Switzerland, July 5-9, 2004, pp. 132136.

[6] G. Moritz et al., "Recent Test Results of the Fast-Pulsed $4 \mathrm{~T} \cos \theta$ Dipole GSI 001," Proc. 2005 Particle Accelerator Conference, Knoxville, TN, USA, May 16-20, 2005. 